\title{
THE VANISHING OF A THETA CONSTANT IS A PECULIAR PHENOMENON
}

\author{
BY H. E. RAUCH ${ }^{1}$ \\ Communicated by E. Calabi, November 28, 1966
}

1. Introduction. The phenomenon in question is the following: (i) if a theta constant $\theta$ vanishes at a point $t$ of Torelli space then its gradient (with respect to the coordinates on Torelli space) vanishes there, too; (ii) on the other hand, the locus $\theta=0$ through $t$ is, generically, a hypersurface with tangent plane defined at $t$, in particular $\theta \not \equiv 0$ on Torelli space.

The reconciliation of (i) and (ii) results from (iii) near $t$ one has $\theta=\Phi^{k}, k>1$ integral, and $\Phi$ analytic with nonvanishing gradient at $t$.

I would speculate that $k=2$, generically, i.e., the locus $\theta=0$ is really the locus $\sqrt{ } \theta=0$.

In the next section I shall prove (i). (ii) is in the thesis of Dr. Farkas [1], while (iii) is an immediate consequence of (i) and (ii) and some standard algebra in several variables. The speculation on the value 2 for $k$ stems from the appearance of those period relations that are known (Schottky). $\S 2$ is a revision of the remarks in [3, pp. 35-37].

2. Definitions and proof of (i). Given a symmetric $g \times g$ complex matrix $A$ with negative definite real part, one can form the Riemann theta function

$$
\theta(u, A)=\sum_{n} \exp (n \cdot A n+2 n \cdot u)
$$

where $n$ ranges over all integral column $g$-vectors, $u$ is a column $g$ vector of complex numbers, and the dot signifies the usual inner product. If, in addition, one is given two column $g$-vectors $\epsilon$ and $\epsilon^{\prime}$ whose entries are 0 or 1 , one defines the first order theta function with binary characteristic

by

$$
\left[\begin{array}{l}
\epsilon \\
\epsilon^{\prime}
\end{array}\right]
$$

$$
\theta\left[\begin{array}{c}
\epsilon \\
\epsilon^{\prime}
\end{array}\right](u, A)=\theta(u+e, A) \exp \left(\frac{\epsilon}{2} \cdot \frac{A \epsilon}{2}+2 \frac{\epsilon \cdot u}{2}+2 \pi i \frac{\epsilon}{2} \cdot \frac{\epsilon^{\prime}}{2}\right) \text {, }
$$

\footnotetext{
${ }^{1}$ Research partially sponsored by the Air Force Office of Scientific Research, Office of Aerospace Research, U. S. Air Force, under AFOSR Grant No. AF-AFOSR$1077-66$.
} 
where $e=\pi i \epsilon^{\prime} / 2+A \epsilon / 2$. Here $e$, and with it

$$
\left[\begin{array}{l}
\epsilon \\
\epsilon^{\prime}
\end{array}\right]
$$

is defined to be even or odd according as $\epsilon \cdot \epsilon^{\prime} \equiv 0,1(\bmod 2)$, and

$$
\theta\left[\begin{array}{l}
\epsilon \\
\epsilon^{\prime}
\end{array}\right](u, A)
$$

is an even or odd function of $u$ according as $e$ is even or odd. In particular if $e$ is even then

$$
\partial \theta\left[\begin{array}{c}
\epsilon \\
\epsilon^{\prime}
\end{array}\right](0, A) / \partial u_{j}=0, \quad j=1, \cdots, g .
$$

Now define the first order theta constant with characteristic

$$
\left[\begin{array}{c}
\epsilon \\
\epsilon^{\prime}
\end{array}\right] \text { by } \theta_{\mathrm{e \epsilon}^{\prime}}=\theta\left[\begin{array}{l}
\epsilon \\
\epsilon^{\prime}
\end{array}\right](0, A) \text {. }
$$

Only the even case is of interest, since all odd theta constants are, of course, identically 0 in $A$.

Let $S$ be a compact Riemann surface of genus $g$ and let $(\gamma, \delta)$ be a canonical homology basis on $S$. Further, let $d \xi_{i}, i=1, \cdots, g$ be a set of abelian differentials of first kind with Riemann's normalization with respect to $(\gamma, \delta)$ :

$$
\int_{\gamma k} d \xi_{h}=\pi i \delta_{h k}
$$

Observe that $d \xi_{j}=\pi i d \zeta_{j}, j=1, \cdots, g$, when the $d \zeta$ are the conventional normal differentials of first kind as defined, e.g., in [3].

Now define, given $P \in S$ fixed and $Q \in S$,

$$
\begin{gathered}
\theta_{\epsilon \epsilon^{\prime}}(Q)=\theta\left[\begin{array}{c}
\epsilon \\
\epsilon^{\prime}
\end{array}\right](u, A), \\
A=\left(a_{i j}\right), \quad a_{i j}=\int_{\delta_{j}} d \xi_{i}, \\
u=\left(\begin{array}{c}
u_{1} \\
\vdots \\
\dot{u_{0}}
\end{array}\right), \quad u_{i}=\int_{P}^{Q} d \xi_{i}, \quad i, j=1, \cdots, g,
\end{gathered}
$$


where the path from $P$ to $Q$ is arbitrary but fixed and the same for each $i$. This is multivalued, but a zero is well defined.

If $e$ is even, then the Riemann vanishing theorem (in view of the vanishing of the first partials of

$$
\theta\left[\begin{array}{l}
\epsilon \\
\epsilon^{\prime}
\end{array}\right](u, A)
$$

at $u=0)([2$, Theorem 6 or 8$])$ implies that

$$
\epsilon_{\epsilon \epsilon^{\prime}}=0 \Rightarrow \epsilon_{\epsilon \epsilon^{\prime}}(Q) \equiv 0 \text {. }
$$

In particular if $Q$ lies in some parameter disk about $P$ and has parameter value $z$ ( $P$ has the value $z=0)$ one finds, setting $P=Q(z=0)$,

$$
0=\frac{d^{2} \theta \epsilon \epsilon^{\prime}(P)}{d z^{2}}=\sum_{i, j} \frac{\partial^{2} \theta\left[\begin{array}{c}
\epsilon \\
\epsilon^{\prime}
\end{array}\right](0, A)}{\partial u_{i} \partial u_{j}} \frac{d \xi_{i}(P)}{d z} \frac{d \xi_{j}(P)}{d z},
$$

where I recall that $P \in S$ is arbitrary.

On the other hand, $S$ and $(\gamma, \delta)$ specify the point $t=\{S, S, 1\} \in J^{o}(S)$ where the transition to any other Torelli space and/or equivalent point on it is easily made by the rules in [3]. I should now like to compute the gradient of $\theta_{c e^{\prime}}$ at $t$. Using Prescription II of [3] with the obvious changes wrought by the substitution of $a_{i j}$ and $d \xi_{i}$ for $\pi_{i j}$ and $d \zeta_{i}$ one finds

$$
\begin{aligned}
\left.\frac{\partial \theta_{\epsilon \epsilon^{\prime}}}{\partial c_{\alpha}}\right|_{c=0} & =-\left.\frac{1}{\pi} \sum_{i \leq j} \frac{\partial \theta_{\epsilon \epsilon^{\prime}}}{\partial a_{i j}} \frac{\partial a_{i j}}{\partial e_{\alpha}}\right|_{c=0} \\
& =-\frac{1}{\pi} \sum_{i \leq j} \frac{\partial \theta\left[\begin{array}{c}
\epsilon \\
\epsilon^{\prime}
\end{array}\right](0, A)}{\partial a_{i j}}\left(\frac{d \xi_{i}}{d z} \frac{d \xi_{j}}{d z}, \mu_{\alpha}\right) \\
& =-\frac{1}{\pi}\left(\sum_{i \leq j} \frac{\partial \theta\left[\begin{array}{c}
\epsilon \\
\epsilon^{\prime}
\end{array}\right](0, A)}{\partial a_{i j}} \frac{d \xi_{j}}{d z} \frac{d \xi_{j}}{d z}, \mu_{\alpha}\right) .
\end{aligned}
$$

But I claim that the sum on the right side of (2) is 4 times the sum in parenthesis in the last expression in (3). If one grants this then (1) and (2) imply immediately that

$$
\theta_{\mathrm{ee}^{\prime}}=\left.0 \Rightarrow \frac{\partial \theta_{\mathrm{ee}}}{\partial c_{\alpha}}\right|_{c=0}=0, \quad \alpha=1, \cdots, 3 g-3,
$$

which is statement (i) of $\S 1$. 
To establish the remaining link I invoke the "heat equations"

$$
\begin{aligned}
2 \frac{\partial \theta\left[\begin{array}{c}
\epsilon \\
\epsilon^{\prime}
\end{array}\right]}{\partial a_{i j}}(u, A) & =\frac{\partial^{2} \theta\left[\begin{array}{l}
\epsilon \\
\epsilon^{\prime}
\end{array}\right]}{\partial u_{i} \partial u_{j}}(u, A), \quad i \neq j, \\
& =\frac{1}{2} \frac{\partial^{2} \theta\left[\begin{array}{l}
\epsilon \\
\epsilon^{\prime}
\end{array}\right]}{\partial u_{i}{ }^{2}}(u, A), \quad i=j,
\end{aligned}
$$

in which I put $u=0$, and then I split the sum $\sum_{i, j}$ in (2) into $2 \sum_{i<j}+\sum_{i=j}$.

\section{BIBLIOGRAPHY}

1. H. Farkas, Special divisors, theta nulls, and analytic subloci of Teichmuiller space, Amer. J. Math. 88 (1966), 881-901.

2. J. Lewittes, Riemann surfaces and the theta function, Acta Math. 111 (1964), $37-61$.

3. H. E. Rauch, $A$ transcendental view of the space of algebraic Riemann surfaces, Bull. Amer. Math. Soc. 71 (1965), 1-39.

Belfer Graduate School of Science, Yeshiva University 